\title{
A simple access to 3,6-branched oligosaccharides: Synthesis of a glycopeptide derivative that relates to Lycium barbarum $\mathbf{L}$.
}

\author{
Yuguo Du,* Meimei Zhang, Feng Yang and Guofeng Gu \\ Research Center for Eco-Environmental Sciences, Chinese Academy of Sciences, PO Box 2871, \\ Beijing 100085, Beijing, China
}

Received (in Cambridge, UK) 17th September 2001, Accepted 17th October 2001

First published as an Advance Article on the web 8th November 2001

\begin{abstract}
An efficient method is described for the synthesis of galactopyranosyl-containing 3,6-branched oligosaccharides using isopropyl thiogalactopyranoside as starting material. This method is successfully applied to the preparation of a glycopeptide derivative that relates to Lycium barbarum $\mathrm{L}$. The potential application of isopropyl thioglycoside in glycosylation is also investigated.
\end{abstract}

\section{Introduction}

The availability of oligosaccharides and their analogues as substrates can provide insight into their biological roles and might lead to the discovery of novel carbohydrate-based therapeutics. ${ }^{1}$ Despite many advances in the chemical synthesis of oligosaccharides, it is still time-consuming and often difficult to synthesize these highly asymmetric and densely functionalized molecules. Among the glycosylation methodologies currently used in carbohydrate chemistry, those using glycosyl trichloroacetimidate and thioglycosides are the most attractive choices for oligosaccharide assembly. ${ }^{2}$ We have recently disclosed a new method for one-pot synthesis of 3,6-differentially protected carbohydrate building blocks and have successfully applied this method to natural oligosaccharides' preparation. ${ }^{3}$ Lycium barbarum L., a famous traditional Chinese herbal medicine, has been widely used in China as a health-protection agent for 2300 years. ${ }^{4}$ We here report the first example of a simple synthesis of a glycopeptide derivative that relates to Lycium barbarum L. using isopropyl thiogalactopyranosides as practical glycosylation building blocks.

\section{Results and discussion}

In our previous communication, ${ }^{3}$ we have described a procedure for one-pot synthesis of 3,6-differentially protected carbohydrate building blocks. Thus, commercially available isopropyl 1-thio- $\beta$-D-galactopyranoside $\mathbf{1}$ was selectively tritylated on the primary hydroxy group with triphenylmethyl chloride $(\mathrm{TrCl})$ in pyridine, silylated with tert-butylchlorodimethylsilane (TBDMSCl) in DMF on C-3 and then benzoylated with benzoyl chloride in one pot to afford isopropyl 2,4-di$O$-benzoyl-3- $O$-tert-butyldimethylsilyl-6- $O$-trityl-1-thio- $\beta$-Dgalactopyranoside 2 in $76 \%$ isolated overall yield (Scheme 1). Treatment of compound $\mathbf{2}$ with $90 \%$ trifluoroacetic acid (TFA) gave 3,6-diol $\mathbf{3}$ in 80\% yield. Interestingly, treatment of $\mathbf{2}$ with $50 \%$ aq. TFA furnished 3-O-silylated product 4 as a major component. It is noteworthy that attempted preparation of the 3,6-diol via acidic hydrolysis of acetylated analogue isopropyl 2,4-di- $O$-acetyl-3- $O$-tert-butyldimethylsilyl-6- $O$-trityl-1-thio- $\beta$ D-galactopyranoside ${ }^{3} 5$ gave exclusively acetyl-migrated product isopropyl 2,6-di- $O$-acetyl-1-thio- $\beta$-D-galactopyranoside $6(85 \%)$. Compared with 3 , chemical shifts of H-6a and H-6b in 6 moved downfield to $\delta 4.26$ and 4.37 from $\delta 3.58$ and 3.77 , respectively, confirming the occurrence of this migration. Coupling reaction of 3,6-diol 3 and 2.1 equiv. of 2,3,4,6- tetra- $O$-benzoyl- $\alpha$-D-galactopyranosyl trichloroacetimidate 7 in anhydrous $\mathrm{CH}_{2} \mathrm{Cl}_{2}$ using TMSOTf as catalyst gave trisaccharide 8 in $90 \%$ yield. No intermolecular aglycone transfer ${ }^{5}$ was observed in this case. Convergently, fully acetylated isopropyl $\beta$-D-galactopyranoside (IPTG) 9 was condensed with L-serine derivative $\mathbf{1 0}^{6}$ in methylene dichloride in the presence of $\mathrm{N}$-iodosuccinimide (NIS) and TMSOTf to afford glycopeptide derivative 11, which was subjected to a sequence of protecting-group manipulations to give desired acceptor $\mathbf{1 4}$ in a total yield of $45 \%$ from $\mathbf{1 0}$. Coupling reaction of $\mathbf{8}$ and $\mathbf{1 4}$ likewise gave glycopeptide $\mathbf{1 5}$ in excellent yield.

Encouraged by the above result, we also tried the coupling reaction of glucosamine derivative $\mathbf{1 6}$ with 3,6-diol 3, and the trisaccharide derivative 17, corresponding to human bloodgroup substrates, was obtained in 53.6\% yield (Scheme 2). The fair yield of this reaction may be ascribed to the quick consumption of imidate $\mathbf{1 6}$ and about $20 \%$ of isopropyl 2,3,4,6-tetra- $O$-benzoyl- $\beta$-D-galactopyranosyl- $(1 \longrightarrow 6)$-2,4-di- $O$ benzoyl-1-thio- $\beta$-D-galactopyranoside was also isolated after separation on a column.

Condensation of fully benzylated isopropyl thioglycoside $\mathbf{1 8}$ and acceptor $19^{7}$ in the presence of NIS and TMSOTf in anhydrous methylene dichloride at $0{ }^{\circ} \mathrm{C}$ achieved $\alpha$-linked disaccharide 20 in $80 \%$ yield. A singlet at $\delta 4.91$ in the ${ }^{1} \mathrm{H}$ NMR spectrum, that was further determined as the signal for $\mathrm{H}-1^{\prime}$ by 2D spectroscopy, confirmed the presence of an $\alpha$ glycosidic linkage in 20. Similar reaction using MeOTf as promoter gave an inseparable mixture of 1,2-cis- and 1,2-trans-linked disaccharides in $56-70 \%$ yield. The yields seem dependent on the amount of MeOTf used in the reactions (we used 2-5 equiv.) and part of the acceptor 19 could be recovered.

The armed-disarmed strategy $^{8}$ was next investigated using isopropyl thioglactopyranoside glycosyl donor $\mathbf{1 8}$ and NIS-TMSOTf as catalyst. When armed donor $\mathbf{1 8}$ was regioselectively glycosylated with disarmed acceptor thioglycoside $21^{9}$ in anhydrous methylene dichloride-diethyl ether $(1: 1, \mathrm{v} / \mathrm{v})$ co-solvent, $\alpha$-linked disaccharide $\mathbf{2 2}$ was obtained in an isolated yield of $62 \%$ after recovery of $60 \mathrm{mg}$ of $\mathbf{2 1}$ (see Experimental section). Glycosylation of $\mathbf{1 8}$ and acceptor $\mathbf{2 3}$ in diethyl ether, on the other hand, furnished disaccharide $\mathbf{2 4}$ in $96 \%$ yield as a mixture of the $\alpha$-and $\beta$-anomer in the ratio of $2: 1$. Reaction of 18 and 25 was not straightforward. This may be caused by the lower activity of 4-OH in $\mathbf{2 5}$.

In summary, we present here a highly efficient and practical method for the preparation of 3,6-branched galactopyranosyl oligosaccharides. This method is successfully applied to the 
synthesis of a glycopeptide derivative that relates to Lycium barbarum L. This is the first report of using isopropyl thioglycosides as glycosyl donors. Its applications in $\alpha$-glycosidic bond formation and the potential usage in the armed-disarmed glycosylation strategy were also investigated.

\section{Experimental}

Optical rotations were determined at $25^{\circ} \mathrm{C}$ with a Perkin-Elmer Model 241-Mc automatic polarimeter and $[\alpha]_{\mathrm{D}^{-}}$-values are in units of $10^{-1} \mathrm{deg}^{2} \mathrm{~cm}^{-1}$. Melting points were determined with

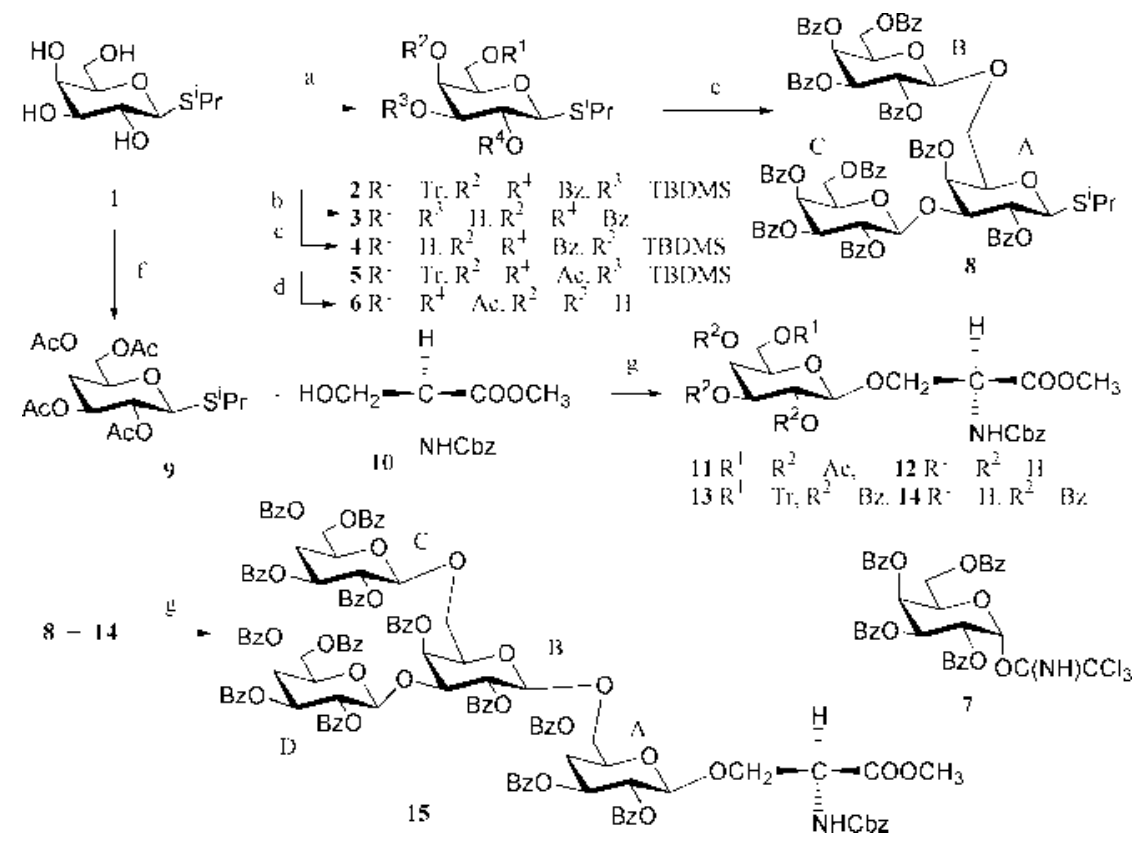

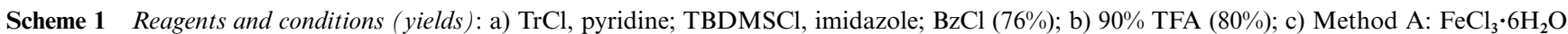
(50\%); Method B: 50\% TFA (86.3\%); d) 90\% TFA (85\%); e) TMSOTf (90\%); f) Ac $\mathrm{A}_{2} \mathrm{O}$, pyridine (100\%); g) NIS, TMSOTf (87\% for $11,91 \%$ for 15 ).

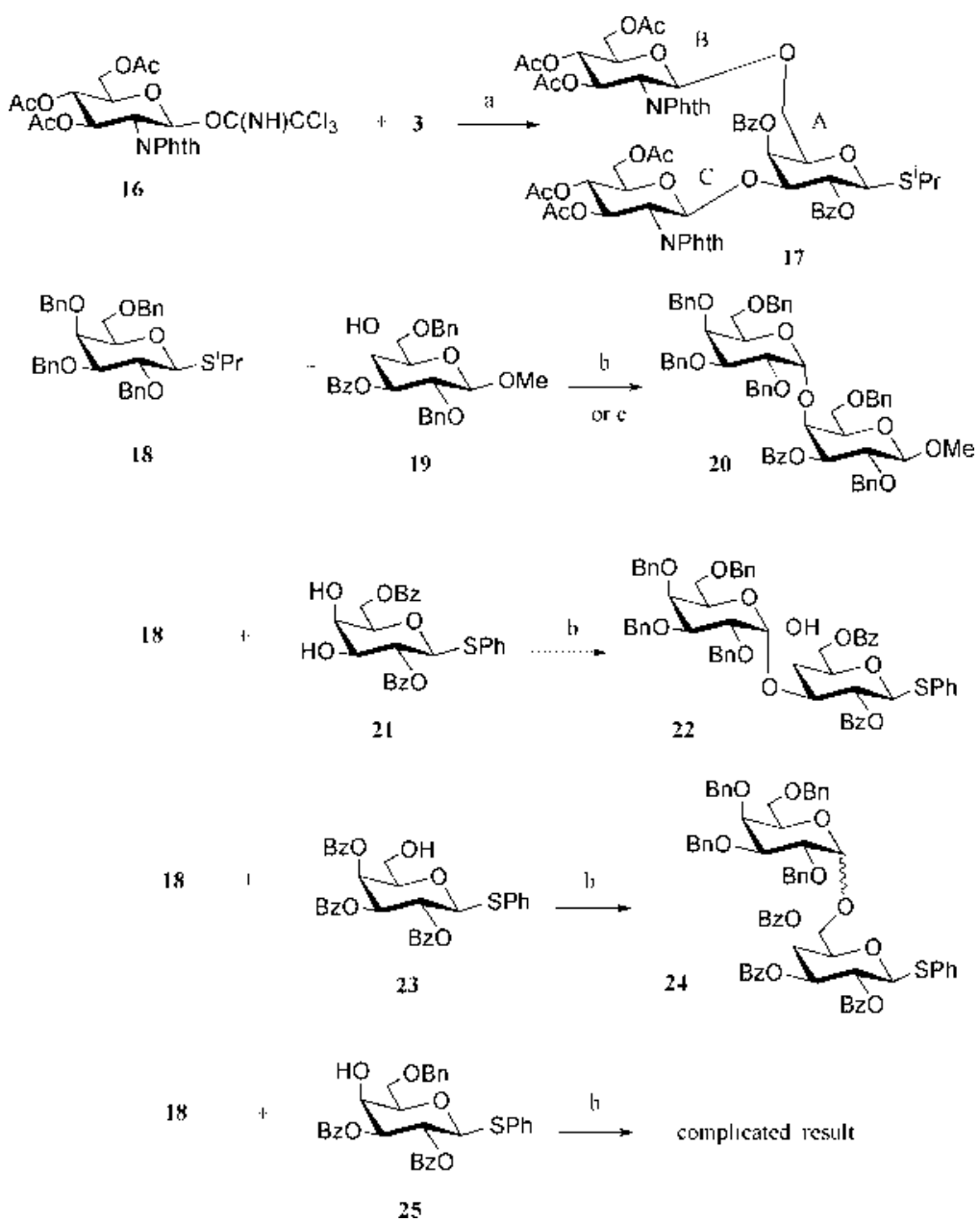

Scheme 2 Reagents and conditions (yields): a) TMSOTf, $\mathrm{CH}_{2} \mathrm{Cl}_{2}(54 \%)$; b) NIS, TMSOTf, $\mathrm{Et}_{2} \mathrm{O}$ ( $80 \%$ for 20, 62\% for 22, 96\% for 24); c) $\mathrm{MeOTf}$, $\mathrm{CH}_{2} \mathrm{Cl}_{2}(56-70 \%)$. 
a 'Mel-Temp' apparatus and are uncorrected. ${ }^{1} \mathrm{H}$ NMR, ${ }^{13} \mathrm{C}$ NMR and ${ }^{1} \mathrm{H}-{ }^{1} \mathrm{H},{ }^{1} \mathrm{H}-{ }^{13} \mathrm{C}$ COSY spectra were recorded with a Bruker ARX 400 spectrometer for solution in $\mathrm{CDCl}_{3}$. Chemical shifts are given in ppm downfield from internal $\mathrm{Me}_{4} \mathrm{Si}$. Mass spectra were measured using MALDI-TOF-MS with $\alpha$-cyano4-hydroxycinnamic acid as matrix. Thin-layer chromatography (TLC) was performed on silica gel $\mathrm{HF}_{254}$ with detection by charring with $30 \%(\mathrm{v} / \mathrm{v}) \mathrm{H}_{2} \mathrm{SO}_{4}$ in $\mathrm{MeOH}$ or in some cases by UV detector. Column chromatography was conducted on columns $(16 \times 240 \mathrm{~mm}, 18 \times 300 \mathrm{~mm}, 35 \times 400 \mathrm{~mm})$ of silica gel (100-200 mesh) using EtOAc-petroleum ether (60-90 $\left.{ }^{\circ} \mathrm{C}\right)$ as eluent. Solutions were concentrated at $<60^{\circ} \mathrm{C}$ under diminished pressure.

\section{Isopropyl 2,4-di-O-benzoyl-3-O-tert-butyldimethylsilyl-6-O- trityl-1-thio- $\beta$-D-galactopyranoside 2}

To a solution of 1 (710 $\mathrm{mg}, 2.98 \mathrm{mmol})$ in pyridine $(2 \mathrm{~mL})$ were added $\mathrm{TrCl}(1.05 \mathrm{~g}, 3.7 \mathrm{mmol})$ and DMAP (20 mg). The mixture was stirred at $80{ }^{\circ} \mathrm{C}$ for $16 \mathrm{~h}$ then cooled to $0{ }^{\circ} \mathrm{C}$. To the above reaction mixture was added imidazole $(408 \mathrm{mg}$, $6.0 \mathrm{mmol})$ in one portion. A solution of TBDMSCl $(450 \mathrm{mg}$, $3.0 \mathrm{mmol})$ in DMF $(3 \mathrm{~mL})$ was added portionwise during $1.5 \mathrm{~h}$. The mixture was stirred at room temperature for $4 \mathrm{~h}$, then premixed benzoyl chloride $(1.05 \mathrm{~g}, 7.46 \mathrm{mmol})$ and pyridine $(1 \mathrm{~mL})$ was added. The reaction mixture was stirred at $50{ }^{\circ} \mathrm{C}$ for $24 \mathrm{~h}$, then poured into ice-cold water, and extracted with EtOAc. The organic phase was concentrated to dryness by coevaporation with toluene. The residue was subjected to column chromatography on silica gel using petroleum ether-EtOAc (3 : 2) as eluent to give $\mathbf{2}(1.65 \mathrm{~g}, 76 \%)$ as a white foam; $[\alpha]_{\mathrm{D}}^{25}+17$ (c $\left.1, \mathrm{CHCl}_{3}\right) ;{ }^{1} \mathrm{H} \mathrm{NMR} \delta-0.12,0.11\left[2 \mathrm{~s}, 6 \mathrm{H}, \mathrm{Si}\left(\mathrm{CH}_{3}\right)_{2}\right], 0.61$ (s, $9 \mathrm{H}, t$-Bu), 1.26, 1.32 [2 d, $\left.6 \mathrm{H}, J 6.8 \mathrm{~Hz}, \mathrm{CH}\left(\mathrm{CH}_{3}\right)_{2}\right], 3.18$ $\left(\mathrm{dd}, 1 \mathrm{H}, J_{5,6 \mathrm{a}} 6.6 \mathrm{~Hz}, J_{6 \mathrm{a}, 6 \mathrm{~b}} 9.6 \mathrm{~Hz}, \mathrm{H}-6 \mathrm{a}\right), 3.22-3.31(\mathrm{~m}, 1 \mathrm{H}$ $\mathrm{SCH}), 3.44$ (dd, $\left.1 \mathrm{H}, J_{5,6 \mathrm{~b}} 6.6 \mathrm{~Hz}, \mathrm{H}-6 \mathrm{~b}\right), 3.80$ (t, $\left.1 \mathrm{H}, \mathrm{H}-5\right), 4.06$ $\left(\mathrm{dd}, 1 \mathrm{H}, J_{2,3} 9.2 \mathrm{~Hz}, J_{3,4} 3.2 \mathrm{~Hz}, \mathrm{H}-3\right), 4.68\left(\mathrm{~d}, 1 \mathrm{H}, J_{1,2} 9.2 \mathrm{~Hz}\right.$, $\mathrm{H}-1), 5.46$ (t, $1 \mathrm{H}, \mathrm{H}-2), 5.71$ (d, $1 \mathrm{H}, \mathrm{H}-4), 7.14-8.17$ (m, $25 \mathrm{H}$, $\mathrm{Ph})\left(\right.$ Calc. for $\mathrm{C}_{48} \mathrm{H}_{54} \mathrm{O}_{7} \mathrm{SSi}$ : C, 71.79; H, 6.78. Found: C, 71.96; $\mathrm{H}, 6.65 \%)$.

\section{Isopropyl 2,4-di- $\boldsymbol{O}$-benzoyl-1-thio- $\boldsymbol{\beta}$-D-galactopyranoside 3}

Compound 2 (1.65 g, $2.07 \mathrm{mmol}$ ) was dissolved in 90\% aq. TFA $(15 \mathrm{~mL})$ and the solution was stirred at room temperature for 2 $\mathrm{h}$. Toluene $(50 \mathrm{~mL})$ was added and the solvents were evaporated in vacuo to give a residue, which was purified by silica gel column chromatography (petroleum ether-EtOAc, $2: 1$ ) to give $3(765 \mathrm{mg}, 80 \%)$ as a syrup; $[\alpha]_{\mathrm{D}}^{25}+15\left(c 1, \mathrm{CHCl}_{3}\right) ;{ }^{1} \mathrm{H}$ NMR $\delta 1.34,1.28\left[2 \mathrm{~d}, 6 \mathrm{H}, J 6.8 \mathrm{~Hz}, \mathrm{CH}\left(\mathrm{CH}_{3}\right)_{2}\right], 3.23-3.30(\mathrm{~m}, 1 \mathrm{H}$, $\mathrm{SCH}), 3.58$ (dd, $\left.1 \mathrm{H}, J_{5,6 \mathrm{a}} 7.2 \mathrm{~Hz}, J_{6 \mathrm{a}, 6 \mathrm{~b}} 12 \mathrm{~Hz}, \mathrm{H}-6 \mathrm{a}\right), 3.77$ (dd, $\left.1 \mathrm{H}, J_{5,6 \mathrm{~b}} 6.4 \mathrm{~Hz}, \mathrm{H}-6 \mathrm{~b}\right), 3.86-3.90(\mathrm{~m}, 1 \mathrm{H}, \mathrm{H}-5), 4.14$ (dd, $1 \mathrm{H}$, $\left.J_{2,3} 10.0 \mathrm{~Hz}, J_{3,4} 3.4 \mathrm{~Hz}, \mathrm{H}-3\right), 4.80$ (d, $\left.1 \mathrm{H}, J_{1,2} 10.0 \mathrm{~Hz}, \mathrm{H}-1\right)$, $5.43(\mathrm{t}, 1 \mathrm{H}, \mathrm{H}-2), 5.62(\mathrm{~d}, 1 \mathrm{H}, \mathrm{H}-4), 7.26-8.14(\mathrm{~m}, 10 \mathrm{H}, \mathrm{Ph})$ (Calc. for $\mathrm{C}_{23} \mathrm{H}_{26} \mathrm{O}_{7} \mathrm{~S}$ : C, 61.87; H, 5.87. Found: C, 61.53; $\mathrm{H}$, $5.91 \%)$.

\section{Isopropyl 2,4-di- $O$-benzoyl-3- $O$-tert-butyldimethylsilyl-1-thio- $\beta$ -} D-galactopyranoside 4

Method A. To a solution of $2(1 \mathrm{~g}, 1.47 \mathrm{mmol})$ in methylene dichloride $(10 \mathrm{~mL})$ was added $\mathrm{FeCl}_{3}$ hexahydrate $(947 \mathrm{mg}$, $3.50 \mathrm{mmol}$ ) and the mixture was stirred at room temperature for $3 \mathrm{~h}$. It was then diluted with $\mathrm{CH}_{2} \mathrm{Cl}_{2}(50 \mathrm{~mL})$ and washed with cold water $(\times 3)$. The water phase was re-extracted with $\mathrm{CH}_{2} \mathrm{Cl}_{2}$ $(20 \mathrm{~mL})$ and the organic phases were combined and concentrated to dryness, and the residue was subjected to silica gel column chromatography (petroleum ether-EtOAc, $2: 1$ ) to give crystalline compound $\mathbf{4}(322 \mathrm{mg}, 50 \%)$.

Method B. To a solution of $2(420 \mathrm{mg}, 0.52 \mathrm{mmol})$ in methylene dichloride $(0.5 \mathrm{~mL})$ was added $50 \%$ aq. TFA $(3 \mathrm{~mL})$.
The mixture was stirred at room temperature for $30 \mathrm{~min}$, then neutralized with sodium bicarbonate and extracted with $\mathrm{CH}_{2} \mathrm{Cl}_{2}(2 \times 20 \mathrm{~mL})$. The organic phases were combined and concentrated to dryness, and the residue was purified on a silica gel column (petroleum ether-EtOAc, $2: 1)$ to give $4(253 \mathrm{mg}$, $86.3 \%)$ as crystals: $\mathrm{mp} 110-112{ }^{\circ} \mathrm{C} ;[a]_{\mathrm{D}}^{25}+76\left(c 1, \mathrm{CHCl}_{3}\right) ;{ }^{1} \mathrm{H}$ NMR $\delta 0.01,0.16\left[2 \mathrm{~s}, 6 \mathrm{H}, \mathrm{Si}\left(\mathrm{CH}_{3}\right)_{2}\right], 0.74(\mathrm{~s}, 9 \mathrm{H}, t-\mathrm{Bu}), 1.41$, $1.44\left[2 \mathrm{~d}, 6 \mathrm{H}, J 6.6 \mathrm{~Hz}, \mathrm{SCH}\left(\mathrm{CH}_{3}\right)_{2}\right], 3.35-3.41$ (m, $\left.1 \mathrm{H}, \mathrm{SCH}\right)$, $3.72\left(\mathrm{dd}, 1 \mathrm{H}, J_{5,6 \mathrm{a}} 6.3 \mathrm{~Hz}, J_{6 \mathrm{a}, 6 \mathrm{~b}} 11.4 \mathrm{~Hz}, \mathrm{H}-6 \mathrm{a}\right), 3.93$ (dd, $1 \mathrm{H}$, $\left.J_{5,6 \mathrm{~b}} 5.7 \mathrm{~Hz}, \mathrm{H}-6 \mathrm{~b}\right), 3.98-4.06(\mathrm{~m}, 1 \mathrm{H}, \mathrm{H}-5), 4.28(\mathrm{dd}, 1 \mathrm{H}$, $\left.J_{2,3} 9.6 \mathrm{~Hz}, J_{3,4}<1.0 \mathrm{~Hz}, \mathrm{H}-3\right), 4.90\left(\mathrm{~d}, 1 \mathrm{H}, J_{1,2} 9.9 \mathrm{~Hz}, \mathrm{H}-1\right)$, $5.63(\mathrm{~d}, 1 \mathrm{H}, \mathrm{H}-4), 5.73$ (t, $1 \mathrm{H}, \mathrm{H}-2), 7.55-8.29(\mathrm{~m}, 10 \mathrm{H}, \mathrm{Ph})$ (Calc. for $\mathrm{C}_{29} \mathrm{H}_{40} \mathrm{O}_{7} \mathrm{SSi}$ : C, 62.11; H, 7.19. Found: C, 62.39; H, $7.05 \%)$.

\section{Isopropyl 2,6-di- $\boldsymbol{O}$-acetyl-1-thio- $\beta$-D-galactopyranoside 6}

Compound $5^{3}$ (200 mg, $0.29 \mathrm{mmol}$ ) was dissolved in $90 \%$ aq. trifluoroacetic acid $(5 \mathrm{~mL})$. The solution was stirred at room temperature for $2 \mathrm{~h}$ and then co-evaporated with toluene to dryness under diminished pressure. Column chromatography using $1: 1$, petroleum ether-EtOAc as eluent gave syrupy 6 $(80 \mathrm{mg}, 85 \%) ;[\alpha]_{\mathrm{D}}^{25}+4\left(c 1, \mathrm{CHCl}_{3}\right) ;{ }^{1} \mathrm{H}$ NMR $\delta 1.30,1.31[2 \mathrm{~d}$, $\left.6 \mathrm{H}, J 5.4 \mathrm{~Hz}, \mathrm{SCH}\left(\mathrm{CH}_{3}\right)_{2}\right], 2.08,2.13\left(2 \mathrm{~s}, 6 \mathrm{H}, 2 \times \mathrm{COCH}_{3}\right)$, 3.16-3.20 (m, 1 H, SCH), 3.65-3.68 (m, 2 H, H-5, H-3), 3.93 (s, $1 \mathrm{H}, \mathrm{H}-4), 4.26\left(\mathrm{dd}, 1 \mathrm{H}, J_{5,6 \mathrm{a}} 6.0 \mathrm{~Hz}, J_{6 \mathrm{a}, 6 \mathrm{~b}} 11.2 \mathrm{~Hz}, \mathrm{H}-6 \mathrm{a}\right)$, 4.37 (dd, $\left.1 \mathrm{H}, J_{5,6 \mathrm{~b}} 5.6 \mathrm{~Hz}, \mathrm{H}-6 \mathrm{~b}\right), 4.48$ (d, $\left.1 \mathrm{H}, J_{1,2} 9.6 \mathrm{~Hz}, \mathrm{H}-1\right)$, $4.98\left(\mathrm{t}, 1 \mathrm{H}, J_{2,3} 11.6 \mathrm{~Hz}, \mathrm{H}-2\right)\left(\right.$ Calc. for $\mathrm{C}_{13} \mathrm{H}_{22} \mathrm{O}_{7} \mathrm{~S}: \mathrm{C}, 48.43$; H, 6.88. Found: C, 48.30; H, 6.99\%).

\section{2,3,4,6-Tetra- $O$-benzoyl- $\alpha$-D-galactopyranosyl trichloro- acetimidate 7}

1,2,3,4,6-Penta- $O$-benzoyl-D-galactopyranose (6 g, $8.57 \mathrm{mmol})$ was dissolved in ammonia-saturated THF-MeOH $(6: 4,100$ $\mathrm{mL}$ ). The mixture was stirred at room temperature for $15 \mathrm{~h}$, then concentrated to dryness, and purified on a silica gel column with $3: 1$, petroleum ether-EtOAc as eluent to yield 2,3,4,6-tetra- $O$-benzoyl-D-galactose ( $4.08 \mathrm{~g}, 80 \%)$. To a solution of 2,3,4,6-tetra- $O$-benzoyl-D-galactose (4 g, $6.71 \mathrm{mmol}$ ) in anhydrous $\mathrm{CH}_{2} \mathrm{Cl}_{2}(25 \mathrm{~mL})$ were added trichloroacetonitrile (2 $\mathrm{mL}, 20 \mathrm{mmol}$ ) and 1,8-diazabicyclo[5.4.0]undec-7-ene (DBU), $(0.45 \mathrm{~mL}, 3.0 \mathrm{mmol})$, and the mixture was stirred at room temperature for $4 \mathrm{~h}$. TLC $(3: 1$, petroleum ether-EtOAc) indicated that the reaction was complete. The mixture was concentrated and purified on a silica gel column using $2: 1$, petroleum ether-EtOAc as eluent to give foamy 7 (4.47 g, 90\%); $[a]_{\mathrm{D}}^{25}+122\left(c 1, \mathrm{CHCl}_{3}\right) ;{ }^{1} \mathrm{H} \mathrm{NMR} \delta 4.49\left(\mathrm{dd}, 1 \mathrm{H}, J_{5,6 \mathrm{a}} 6.0 \mathrm{~Hz}\right.$, $\left.J_{6 \mathrm{a}, 6 \mathrm{~b}} 11.2 \mathrm{~Hz}, \mathrm{H}-6 \mathrm{a}\right), 4.66$ (dd, $1 \mathrm{H}, J_{5,6 \mathrm{~b}} 6.9 \mathrm{~Hz}, J_{6 \mathrm{a}, 6 \mathrm{~b}} 11.2 \mathrm{~Hz}$, $\mathrm{H}-6 \mathrm{~b}), 4.92$ (dd, $1 \mathrm{H}, \mathrm{H}-5), 6.03$ (dd, $1 \mathrm{H}, J_{1,2} 3.6 \mathrm{~Hz}, J_{2,3} 10.5$ $\mathrm{Hz}, \mathrm{H}-2), 6.14$ (dd, $\left.1 \mathrm{H}, J_{3,4} 2.6 \mathrm{~Hz}, \mathrm{H}-3\right), 6.22$ (d, $\left.1 \mathrm{H}, \mathrm{H}-4\right)$, 6.98 (d, $1 \mathrm{H}, \mathrm{H}-1), 7.22-8.13$ (m, $20 \mathrm{H}, \mathrm{Ph}), 8.69$ (s, 1 H, NH) [MALDI-TOF-MS Calc. for $\mathrm{C}_{36} \mathrm{H}_{28} \mathrm{Cl}_{3} \mathrm{NNaO}_{10}(\mathrm{M}+\mathrm{Na})^{+}$: 762. Found: $\left.m / z, 762(\mathrm{M}+\mathrm{Na})^{+}\right]$.

\section{Isopropyl $O$-[2,3,4,6-tetra- $O$-benzoyl- $\beta$-D-galactopyranosyl- $(1 \rightarrow 6)]-O$-[2,3,4,6-tetra- $O$-benzoyl- $\beta$-D-galactopyranosyl- $(1 \longrightarrow 3)$ ]-2,4-di- $O$-benzoyl-1-thio- $\beta$-D-galactopyranoside 8}

To a cooled solution $\left(-15^{\circ} \mathrm{C}\right)$ of $\mathbf{3}(300 \mathrm{mg}, 0.67 \mathrm{mmol})$ and 7 $(1.06 \mathrm{~g}, 1.43 \mathrm{mmol})$ in dry $\mathrm{CH}_{2} \mathrm{Cl}_{2}(5 \mathrm{~mL})$ was added TMSOTf $(12 \mu \mathrm{L}, 0.06 \mathrm{mmol})$ under $\mathrm{N}_{2}$, and the mixture was stirred at this temperature for $2 \mathrm{~h}$, then neutralized with triethylamine. The reaction mixture was concentrated to a residue, which was purified by silica gel column chromatography (petroleum etherEtOAc, $2: 1)$ to give $8(966 \mathrm{mg}, 90 \%)$ as a white foam; $[a]_{\mathrm{D}}^{25}+14$ (c 1, $\left.\mathrm{CHCl}_{3}\right) ;{ }^{1} \mathrm{H}$ NMR $\delta 1.01,1.13[2 \mathrm{~d}, 6 \mathrm{H}, J 6.5 \mathrm{~Hz}$, $\left.\mathrm{SCH}\left(\mathrm{CH}_{3}\right)_{2}\right], 2.97-3.01(\mathrm{~m}, 1 \mathrm{H}, \mathrm{SCH}), 3.88(\mathrm{dd}, 1 \mathrm{H}, J 8.4 \mathrm{~Hz}$, $10.8 \mathrm{~Hz}, \mathrm{H}-6 \mathrm{a}), 4.04-4.06$ (m, 1 H, H-6), 4.19-4.49 (m, $5 \mathrm{H})$, $4.50(\mathrm{dd}, 1 \mathrm{H}, J 6.0 \mathrm{~Hz}, 11.4 \mathrm{~Hz}, \mathrm{H}-6), 4.64\left(\mathrm{~d}, 1 \mathrm{H}, J_{1,2} 10.2 \mathrm{~Hz}\right.$, 
H-1 $\left.{ }^{\mathrm{A}}\right), 4.69-4.63$ (m, 1 H, H-6), 4.75 (dd, $1 \mathrm{H}, J 5.7 \mathrm{~Hz}, 10.8 \mathrm{~Hz}$ H-6), 5.00 (dd, $\left.1 \mathrm{H}, J 7.8,9.6 \mathrm{~Hz}, \mathrm{H}-2^{\mathrm{A}}\right), 5.00(\mathrm{~d}, 1 \mathrm{H}, J 7.8 \mathrm{~Hz}$, $\left.\mathrm{H}-1^{\mathrm{C}}\right), 5.43\left(\mathrm{dd}, 1 \mathrm{H}, J 10.5,3.3 \mathrm{~Hz}, \mathrm{H}-3^{\mathrm{C}}\right), 5.61$ (distorted t, $1 \mathrm{H}, J$ 8.7, $\left.9.9 \mathrm{~Hz}, \mathrm{H}-2^{\mathrm{B}}\right), 5.58-5.64\left(\mathrm{~m}, 2 \mathrm{H}, \mathrm{H}-3, \mathrm{H}-1^{\mathrm{B}}\right), 5.91$ (distorted t, $\left.1 \mathrm{H}, J 9.0,9.6 \mathrm{~Hz}, \mathrm{H}-2^{\mathrm{C}}\right), 5.92(\mathrm{~d}, 1 \mathrm{H}, J 3.0 \mathrm{~Hz}$, $\left.\mathrm{H}-4^{\mathrm{B}}\right), 5.98\left(\mathrm{~d}, 1 \mathrm{H}, J 3.0 \mathrm{~Hz}, \mathrm{H}-4^{\mathrm{C}}\right), 6.06(\mathrm{~d}, 1 \mathrm{H}, J 3.3 \mathrm{~Hz}$ $\left.\mathrm{H}-4^{\mathrm{A}}\right), 7.05-8.23(\mathrm{~m}, 50 \mathrm{H}, \mathrm{Ph})\left(\mathrm{Calc}\right.$. for $\mathrm{C}_{91} \mathrm{H}_{78} \mathrm{O}_{25} \mathrm{~S}: \mathrm{C}, 68.16$; $\mathrm{H}, 4.90$. Found: C, 68.01; H, 5.02\%).

\section{Isopropyl 2,3,4,6-tetra- $O$-acetyl-1-thio- $\beta$-D-galactopyranoside 9}

Standard acetylation of IPTG $(2.0 \mathrm{~g}, 8.4 \mathrm{mmol})$ with acetic anhydride $(8 \mathrm{~mL})$ in pyridine $(10 \mathrm{~mL})$ gave a quantitative yield of crystalline 9, mp 70-72 ${ }^{\circ} \mathrm{C} ;{ }^{1} \mathrm{H}$ NMR $\delta 1.31,1.32$ [2 d, $6 \mathrm{H}$, $\left.\mathrm{CH}\left(\mathrm{CH}_{3}\right)_{2}\right], 1.99,2.04,2.06,2.15(4 \mathrm{~s}, 12 \mathrm{H}, 4 \times \mathrm{Ac}), 3.19[\mathrm{~m}$, $\left.1 \mathrm{H}, \mathrm{CH}\left(\mathrm{CH}_{3}\right)_{2}\right], 3.93$ (br t, $\left.1 \mathrm{H}, J_{5,6 \mathrm{a}} 6.4, J_{5,6 \mathrm{~b}} 6.9 \mathrm{~Hz}, \mathrm{H}-5\right), 4.10$ (dd, $\left.1 \mathrm{H}, J_{6 \mathrm{a}, 6 \mathrm{~b}} 11.3 \mathrm{~Hz}, \mathrm{H}-6 \mathrm{a}\right), 4.18$ (dd, $\left.1 \mathrm{H}, \mathrm{H}-6 \mathrm{~b}\right), 4.58$ (d, $\left.1 \mathrm{H}, J_{1,2} 10.0 \mathrm{~Hz}, \mathrm{H}-1\right), 5.06\left(\mathrm{dd}, 1 \mathrm{H}, J_{3,4} 3.6 \mathrm{~Hz}, \mathrm{H}-3\right), 5.22(\mathrm{t}$, $\left.1 \mathrm{H}, J_{2,3} 10.0 \mathrm{~Hz}, \mathrm{H}-2\right), 5.43(\mathrm{~d}, 1 \mathrm{H}, \mathrm{H}-4)\left(\right.$ Calc. for $\mathrm{C}_{17} \mathrm{H}_{26} \mathrm{O}_{9} \mathrm{~S}$ : C, 50.24; H, 6.45. Found: C, 50.37; H, 6.55\%).

\section{$O$-(2,3,4,6-Tetra- $O$-acetyl- $\beta$-D-galactopyranosyl)- $N$-benzyloxy-} carbonyl-L-serine methyl ester 11

To a pre-cooled solution $\left(0{ }^{\circ} \mathrm{C}\right)$ of compound $\mathbf{1 0}^{6}(708 \mathrm{mg}$, $2.8 \mathrm{mmol})$ and $9(1.2 \mathrm{~g}, 2.95 \mathrm{mmol})$ in $\mathrm{CH}_{2} \mathrm{Cl}_{2}(20 \mathrm{~mL})$ were added NIS (900 mg, $4 \mathrm{mmol})$ and TMSOTf $(90 \mu \mathrm{L}, 0.5 \mathrm{mmol})$ under a nitrogen atmosphere. The mixture was stirred at this temperature for $2 \mathrm{~h}$, TLC $(2: 1$, petroleum ether-EtOAc) showed the starting material had disappeared. The reaction mixture was quenched with $\mathrm{Et}_{3} \mathrm{~N}$, then concentrated, and the residue was subjected to flash chromatography with 2 : 1 , petroleum ether-EtOAc as eluent to give $11(1.42 \mathrm{~g}, 87 \%)$ as a syrup; $[\alpha]_{\mathrm{D}}^{25}+6\left(c 1, \mathrm{CHCl}_{3}\right) ;{ }^{1} \mathrm{H}$ NMR $\delta 1.95,2.00,2.01,2.11$ $(4 \mathrm{~s}, 4 \times 3 \mathrm{H}, 4 \times \mathrm{Ac}), 3.74\left(\mathrm{~s}, 3 \mathrm{H}, \mathrm{OCH}_{3}\right), 3.76-3.82(\mathrm{~m}, 3 \mathrm{H}$ H-5, H-6a, H-6b), 3.84-3.88 (m, $1 \mathrm{H}$, one proton of $\left.\mathrm{OCH}_{2}\right)$, $4.20-4.24\left(\mathrm{~m}, 1 \mathrm{H}\right.$, other proton of $\left.\mathrm{OCH}_{2}\right), 4.41\left(\mathrm{~d}, 1 \mathrm{H}, J_{1,2} 7.8\right.$ $\mathrm{Hz}, \mathrm{H}-1), 4.44-4.46(\mathrm{~m}, 1 \mathrm{H}, \mathrm{OCH}), 4.94\left(\mathrm{dd}, 1 \mathrm{H}, J_{2,3} 10.2 \mathrm{~Hz}\right.$ $\left.J_{3,4} 3.3 \mathrm{~Hz}, \mathrm{H}-3\right), 5.11-5.14\left(\mathrm{~m}, 3 \mathrm{H}, \mathrm{OCH}_{2}, \mathrm{H}-2\right), 5.33(\mathrm{~d}, 1 \mathrm{H}$, $\left.J_{3,4} 3.3 \mathrm{~Hz}, \mathrm{H}-4\right), 5.55(\mathrm{~d}, 1 \mathrm{H}, J 8.1 \mathrm{~Hz}, \mathrm{NH}), 7.23-7.33(\mathrm{~m}, 5 \mathrm{H}$, $\mathrm{Ph})\left(\right.$ Calc. for $\mathrm{C}_{26} \mathrm{H}_{33} \mathrm{NO}_{14}: \mathrm{C}, 53.51 ; \mathrm{H}, 5.70$. Found: C, 53.69; $\mathrm{H}, 5.61 \%)$.

\section{$O$-(2,3,4-Tri- $O$-benzoyl-6- $O$-trityl- $\beta$-D-galactopyranosyl)- $N$ -} benzyloxycarbonyl-L-serine methyl ester 13

A solution of $11(3.2 \mathrm{~g}, 5.5 \mathrm{mmol})$ in $\mathrm{MeOH}(50 \mathrm{~mL})$ was treated with $\mathrm{NaOMe}(2.5 \mathrm{~mL} ; 0.5 \mathrm{M}$ in $\mathrm{MeOH})$ at room temperature for $4 \mathrm{~h}$. The mixture was neutralized with Amberlite $120\left(\mathrm{H}^{+}\right)$resin, then filtered, and the filtrate was evaporated to give $O$ - $(\beta$-D-galactopyranosyl)- $N$-benzyloxycarbonyl-L-serine methyl ester $12(2.2 \mathrm{~g})$ as a syrup. Compound $\mathbf{1 2}(2.2 \mathrm{~g}$, $5.3 \mathrm{mmol})$ was dissolved in pyridine $(10 \mathrm{~mL})$, and trityl chloride $(2.57 \mathrm{~g}, 9.54 \mathrm{mmol})$ and DMAP $(20 \mathrm{mg})$ were added. The mixture was stirred at room temperature for $48 \mathrm{~h}$, then cooled to $0{ }^{\circ} \mathrm{C}$. To the above reaction mixture was added premixed benzoyl chloride $(2.2 \mathrm{~mL}, 18.5 \mathrm{mmol})$ and pyridine $(3 \mathrm{~mL})$, and the mixture was stirred vigorously at room temperature for $48 \mathrm{~h}$, and then poured into ice-cold water, and extracted with $\mathrm{CH}_{2} \mathrm{Cl}_{2}(2 \times 50 \mathrm{~mL})$. The organic phase was concentrated to dryness by repeated co-evaporation with toluene. The residue was subjected to column chromatography on silica gel with toluene-petroleum ether-EtOAc as eluent $(0.2: 1.5: 1)$ to give $13(3.1 \mathrm{~g}, 58 \%) ;[\alpha]_{\mathrm{D}}^{25}+8\left(c 1, \mathrm{CHCl}_{3}\right) ;{ }^{1} \mathrm{H} \mathrm{NMR} \delta 3.24(\mathrm{dd}, 1 \mathrm{H}$, $\left.J_{5,6 \mathrm{a}} 8.7, J_{6 \mathrm{a}, 6 \mathrm{~b}} 12 \mathrm{~Hz}, \mathrm{H}-6 \mathrm{a}\right), 3.42$ (dd, $\left.1 \mathrm{H}, J_{5,6 \mathrm{~b}} 5.7 \mathrm{~Hz}, \mathrm{H}-6 \mathrm{~b}\right)$, $3.57\left(\mathrm{~s}, 3 \mathrm{H}, \mathrm{OCH}_{3}\right), 3.87-3.91\left(\mathrm{~m}, 1 \mathrm{H}\right.$, one proton of $\left.\mathrm{OCH}_{2}\right)$, $3.96\left(\mathrm{dd}, 1 \mathrm{H}, J_{5,6 \mathrm{a}} 7.5, J_{5,6 \mathrm{~b}} 6.0 \mathrm{~Hz}, \mathrm{H}-5\right), 4.28-4.30(\mathrm{~m}, 1 \mathrm{H}$, other proton of $\left.\mathrm{OCH}_{2}\right), 4.40-4.43(\mathrm{~m}, 1 \mathrm{H}, \mathrm{OCH}), 4.67(\mathrm{~d}, 1 \mathrm{H}$, $\left.J_{1,2} 7.2 \mathrm{~Hz}, \mathrm{H}-1\right), 4.96,5.04\left(2 \mathrm{~d}, 2 \mathrm{H}, J 12.3 \mathrm{~Hz}, \mathrm{OCH}_{2}\right), 5.44(\mathrm{~d}$, $1 \mathrm{H}, J 7.5 \mathrm{~Hz}, \mathrm{NH}), 5.56-5.62(\mathrm{~m}, 2 \mathrm{H}, \mathrm{H}-2, \mathrm{H}-3), 6.02(\mathrm{~s}, 1 \mathrm{H}$,
H-4), 7.05-8.05 (m, 35 H, Ph) (Calc. for $\mathrm{C}_{58} \mathrm{H}_{51} \mathrm{NO}_{13}$ : C, 71.81; $\mathrm{H}, 5.30$. Found: $\mathrm{C}, 71.57$; H, 5.38\%).

\section{$O$-(2,3,4-Tri- $O$-benzoyl- $\beta$-D-galactopyranosyl)- $N$-benzyloxy-} carbonyl-L-serine methyl ester 14

To a solution of 13 (600 mg, $0.62 \mathrm{mmol})$ in methylene dichloride $(10 \mathrm{~mL})$ was added $\mathrm{FeCl}_{3}$ hexahydrate $(406 \mathrm{mg}, 1.5 \mathrm{mmol})$. The mixture was stirred at room temperature for $3 \mathrm{~h}$ and then diluted with more $\mathrm{CH}_{2} \mathrm{Cl}_{2}(50 \mathrm{~mL})$. The above mixture was washed with cold water three times and the combined water phase was re-extracted with $\mathrm{CH}_{2} \mathrm{Cl}_{2}(30 \mathrm{~mL})$. The organic phase were combined and concentrated to dryness, and the residue was subjected to silica gel column chromatography (petroleum ether-EtOAc, $3: 2)$ to give $\mathbf{1 4}(400 \mathrm{mg}, 89 \%)$ as an amorphous solid; $[a]_{\mathrm{D}}^{25}+1\left(c 1, \mathrm{CHCl}_{3}\right) ;{ }^{1} \mathrm{H}$ NMR $\delta 3.35\left(\mathrm{~s}, 3 \mathrm{H}, \mathrm{OCH}_{3}\right)$, 3.56-3.62 (m, $1 \mathrm{H}, \mathrm{H}-5), 3.77$ (dd, $1 \mathrm{H}, J_{5,6 \mathrm{a}} 7.2 \mathrm{~Hz}, J_{6 \mathrm{a}, 6 \mathrm{~b}} 11.7$ Hz, H-6a), 3.99 (dd, 1 H, $\left.J_{5,6 \mathrm{~b}} 5.7 \mathrm{~Hz}, \mathrm{H}-6 \mathrm{~b}\right), 4.09-4.12$ (m, $2 \mathrm{H}$, $\left.\mathrm{OCH}_{2}\right), 4.50-4.53(\mathrm{~m}, 1 \mathrm{H}, \mathrm{OCH}), 4.74\left(\mathrm{~d}, 1 \mathrm{H}, J_{1,2} 7.8 \mathrm{~Hz}\right.$, $\mathrm{H}-1), 5.02$ (d, $1 \mathrm{H}, J 8.4 \mathrm{~Hz}, \mathrm{NH}), 5.09$ (s, $\left.2 \mathrm{H}, \mathrm{OCH}_{2}\right), 5.51-$ 5.55 (m, $2 \mathrm{H}, \mathrm{H}-2, \mathrm{H}-3$ ), 5.73 (br s, $1 \mathrm{H}, \mathrm{H}-4), 7.20-8.09$ (m, 20 $\mathrm{H}, \mathrm{Ph}$ ) (Calc. for $\mathrm{C}_{39} \mathrm{H}_{37} \mathrm{NO}_{13}: \mathrm{C}, 64.37 ; \mathrm{H}, 5.12$. Found: $\mathrm{C}$, $64.25 ; \mathrm{H}, 5.20 \%)$.

$O$ - $\{2,3,4,6$-Tetra- $O$-benzoyl- $\beta$-D-galactopyranosyl- $(1 \longrightarrow 6)-$ $[2,3,4,6$-tetra- $O$-benzoyl- $\beta$-D-galactopyranosyl-( $1 \longrightarrow 3)]-2,4-d i-$ $O$-benzoyl- $\beta$-D-galactopyranosyl-( $\longrightarrow 6)$-2,3,4-tri- $O$-benzoyl- $\beta$ D-galactopyranosyl) $\}$ - $N$-benzyloxycarbonyl-L-serine methyl ester 15

A solution of compound $8(368 \mathrm{mg}, 0.23 \mathrm{mmol})$ and $\mathbf{1 4}$ (153 mg, $0.21 \mathrm{mmol})$ in anhydrous $\mathrm{CH}_{2} \mathrm{Cl}_{2}(3 \mathrm{~mL})$ was treated with NIS (140 mg, $0.62 \mathrm{mmol})$ and TMSOTf ( $37 \mu \mathrm{L}, 0.2 \mathrm{mmol})$ under $\mathrm{N}_{2}$ at $0{ }^{\circ} \mathrm{C}$. The mixture was stirred under these conditions for $2 \mathrm{~h}$, then neutralized with $\mathrm{Et}_{3} \mathrm{~N}$ and concentrated. Column chromatography ( $2: 1$, petroleum ether-EtOAc) of the residue gave glycopeptide $\mathbf{1 5}(430 \mathrm{mg}, 91 \%)$ as a syrup; $[a]_{\mathrm{D}}^{25}+87\left(c 1, \mathrm{CHCl}_{3}\right) ;{ }^{1} \mathrm{H}$ NMR $\delta 3.50\left(\mathrm{~s}, 3 \mathrm{H}, \mathrm{OCH}_{3}\right), 3.50-$ 3.65 (m, $\left.3 \mathrm{H}, \mathrm{H}-5, \mathrm{H}_{2}-6\right)$, 3.89-3.95 (m, $4 \mathrm{H}, \mathrm{H}_{2}-6, \mathrm{H}-5$, one proton of $\left.\mathrm{OCH}_{2}\right), 4.08-4.11\left(\mathrm{~m}, 2 \mathrm{H}, \mathrm{H}-3^{\mathrm{B}}\right.$, other proton of $\left.\mathrm{OCH}_{2}\right), 4.24-4.40(\mathrm{~m}, 5 \mathrm{H}, 3 \times \mathrm{H}-6,2 \times \mathrm{H}-5), 4.53(\mathrm{~d}, 1 \mathrm{H}, J 8.0$ $\left.\mathrm{Hz}, \mathrm{H}-1^{\mathrm{A}}\right), 4.52-4.55\left(\mathrm{~m}, 2 \mathrm{H}, \mathrm{H}-1^{\mathrm{B}}, \mathrm{OCH}\right), 4.70$ (dd, $1 \mathrm{H}, J 6.4$, $11.4 \mathrm{~Hz}, \mathrm{H}-6 \mathrm{~b}), 4.80$ (d, $\left.1 \mathrm{H}, J 7.6 \mathrm{~Hz}, \mathrm{H}^{-1}{ }^{\mathrm{D}}\right), 4.93$ (d, $1 \mathrm{H}, J_{1,2}$ $\left.8.8 \mathrm{~Hz}, \mathrm{H}-1^{\mathrm{C}}\right), 4.94,5.01\left(2 \mathrm{~d}, 2 \mathrm{H}, J 12.4 \mathrm{~Hz}, \mathrm{OCH}_{2}\right), 5.37$ $(\mathrm{d}, 1 \mathrm{H}, J 7.8 \mathrm{~Hz}, \mathrm{NH}), 5.37\left(\mathrm{dd}, 1 \mathrm{H}, J 9.2,3.2 \mathrm{~Hz}, \mathrm{H}-3^{\mathrm{D}}\right), 5.42$ $\left(\mathrm{dd}, 1 \mathrm{H}, J 9.6,3.2 \mathrm{~Hz}, \mathrm{H}-3^{\mathrm{A}}\right), 5.51-5.55\left(\mathrm{~m}, 3 \mathrm{H}, \mathrm{H}-2^{\mathrm{A}}, \mathrm{H}-2^{\mathrm{B}}\right.$, $\left.\mathrm{H}-2^{\mathrm{D}}\right), 5.64\left(\mathrm{dd}, 1 \mathrm{H}, J 9.6,3.2 \mathrm{~Hz}, \mathrm{H}-3^{\mathrm{C}}\right), 5.70(\mathrm{~d}, 1 \mathrm{H}, J 3.2 \mathrm{~Hz}$, $\left.\mathrm{H}-4^{\mathrm{A}}\right),\left(5.75\left(\mathrm{dd}, 1 \mathrm{H}, J 8.0,9.2 \mathrm{~Hz}, \mathrm{H}-2^{\mathrm{C}}\right), 5.84(\mathrm{~d}, 1 \mathrm{H}, J 3.2\right.$ $\left.\mathrm{Hz}, \mathrm{H}-4^{\mathrm{D}}\right), 5.89\left(\mathrm{~d}, 1 \mathrm{H}, J 2.8 \mathrm{~Hz}, \mathrm{H}-4^{\mathrm{B}}\right), 5.99(\mathrm{~d}, 1 \mathrm{H}, J 3.2 \mathrm{~Hz}$, $\left.\mathrm{H}-4^{\mathrm{C}}\right), 7.03-8.15$ (m, $\left.70 \mathrm{H}, \mathrm{Ph}\right) ;{ }^{13} \mathrm{C}$ NMR (100 MHz; $\mathrm{CDCl}_{3}$ ) $\delta 170.00,165.95,165.92,165.86,165.54,165.44(2 \mathrm{C}), 165.36$, 165.19 (2 C), 165.15 (2 C), 164.39 (2 C), 154 (BnOCO), 100.55, $101.40,101.40,101.65$ (4 C-1), 73.95, 72.89, 71.62, 71.48, $71.29,71.04,70.92,70.61,69.87,69.49,69.35,68.63,68.31$, $68.12,67.55,66.88,66.69,61.93,61.46,54.12,53.74,52.46$. [MALDI-TOF-MS Calc. for $\mathrm{C}_{127} \mathrm{H}_{107} \mathrm{NNaO}_{38}(\mathrm{M}+\mathrm{Na})^{+}$: 2276.6. Found: $m / z, 2276.9(\mathrm{M}+\mathrm{Na})^{+}$] (Calc. for $\mathrm{C}_{127} \mathrm{H}_{107^{-}}$ $\mathrm{NO}_{38}$ : C, 67.64; H, 4.78. Found: $\left.\mathrm{C}, 67.85 ; \mathrm{H}, 4.70 \%\right)$.

\section{Isopropyl $O$-[3,4,6-tri- $O$-acetyl-2-deoxy-2-phthalimido- $\beta$-D- glucopyranosyl-(1 $\rightarrow 6)]-O$-[3,4,6-tri- $O$-acetyl-2-deoxy-2- phthalimido- $\beta$-D-glucopyranosyl-( $1 \longrightarrow 3)$ ]-2,4-di- $O$-benzoyl-1- thio- $\beta$-D-galactopyranoside 17}

To a mixture of $\mathbf{3}$ (200 mg, $0.45 \mathrm{mmol}), \mathbf{1 6}(577 \mathrm{mg}, 1.00 \mathrm{mmol})$ and $4 \AA$ molecular sieves in anhydrous $\mathrm{CH}_{2} \mathrm{Cl}_{2}(8 \mathrm{~mL})$ was added TMSOTf $(12 \mu \mathrm{L}, 0.06 \mathrm{mmol})$ under $\mathrm{N}_{2}$ at $-15{ }^{\circ} \mathrm{C}$. The mixture was stirred these conditions for $2 \mathrm{~h}$, at the end of which time TLC $(1: 1$, petroleum ether-EtOAc) indicated that starting material 16 had disappeared. The reaction mixture was neutralized with $\mathrm{Et}_{3} \mathrm{~N}$, then filtered, and the filtrate was 
concentrated. The residue was purified by silica gel column chromatography (petroleum ether-EtOAc, $1: 1)$ to give 17 (308 $\mathrm{mg}, 53.6 \%)$ as a syrup; $[a]_{\mathrm{D}}^{25}+58\left(c 1, \mathrm{CHCl}_{3}\right) ;{ }^{1} \mathrm{H}$ NMR $\delta 0.91$, 0.99 [2 d, $\left.6 \mathrm{H}, \mathrm{CH}\left(\mathrm{CH}_{3}\right)_{2}\right], 1.69,1.83(2 \mathrm{~s}, 6 \mathrm{H}, 2 \times \mathrm{Ac}), 1.96$ $(\mathrm{s}, 6 \mathrm{H}, 2 \times \mathrm{Ac}), 2.03,2.15(2 \mathrm{~s}, 6 \mathrm{H}, 2 \times \mathrm{Ac}), 2.79-2.87(\mathrm{~m}, 1 \mathrm{H}$, $\mathrm{SCH}), 3.56\left(\mathrm{dd}, 1 \mathrm{H}, J 8.6,10.8 \mathrm{~Hz}, \mathrm{H}-6 \mathrm{a}^{\mathrm{A}}\right), 3.75(\mathrm{~m}, 1 \mathrm{H}$ $\left.\mathrm{H}-5^{\mathrm{A}}\right), 3.85-3.91\left(\mathrm{~m}, 2 \mathrm{H}, \mathrm{H}-3^{\mathrm{A}}, \mathrm{H}-6 \mathrm{~b}^{\mathrm{A}}\right), 4.00-4.09(\mathrm{~m}, 2 \mathrm{H}$ $\left.\mathrm{H}-5^{\mathrm{B}}, \mathrm{H}-6 \mathrm{a}^{\mathrm{B}}\right), 4.12\left(\mathrm{dd}, 1 \mathrm{H}, J 8.4 \mathrm{~Hz}, \mathrm{H}-2^{\mathrm{C}}\right), 4.13-4.25(\mathrm{~m}, 3 \mathrm{H}$ $\left.\mathrm{H}-6 \mathrm{~b}^{\mathrm{B}}, \mathrm{H}-5^{\mathrm{C}}, \mathrm{H}-6 \mathrm{a}^{\mathrm{C}}\right), 4.29\left(\mathrm{dd}, 1 \mathrm{H}, J 8.5 \mathrm{~Hz}, \mathrm{H}-2^{\mathrm{B}}\right), 4.35(\mathrm{dd}$, $\left.1 \mathrm{H}, J 9.6,12.6 \mathrm{~Hz}, \mathrm{H}-6 \mathrm{~b}^{\mathrm{C}}\right), 4.52\left(\mathrm{~d}, 1 \mathrm{H}, J 10.1 \mathrm{~Hz}, \mathrm{H}-1^{\mathrm{A}}\right), 5.02$ $\left(\mathrm{t}, 1 \mathrm{H}, J 10.5 \mathrm{~Hz}, \mathrm{H}-4^{\mathrm{C}}\right), 5.16\left(\mathrm{t}, 1 \mathrm{H}, J 10.5 \mathrm{~Hz}, \mathrm{H}-4^{\mathrm{B}}\right), 5.35$ $\left(\mathrm{t}, 1 \mathrm{H}, J 10.1 \mathrm{~Hz}, \mathrm{H}-2^{\mathrm{A}}\right), 5.40\left(\mathrm{~d}, 1 \mathrm{H}, J 8.5 \mathrm{~Hz}, \mathrm{H}-1^{\mathrm{B}}\right), 5.51$ $\left(\mathrm{d}, 1 \mathrm{H}, J 8.4 \mathrm{~Hz}, \mathrm{H}-1^{\mathrm{C}}\right), 5.55\left(\mathrm{dd}, 1 \mathrm{H}, \mathrm{H}-3^{\mathrm{C}}\right), 5.63(\mathrm{~d}, 1 \mathrm{H}, J 3.4$ $\left.\mathrm{Hz}, \mathrm{H}-4^{\mathrm{A}}\right), 5.72\left(\mathrm{dd}, 1 \mathrm{H}, \mathrm{H}-3^{\mathrm{B}}\right), 7.22-8.02(\mathrm{~m}, 18 \mathrm{H}, \mathrm{Ph})$ (Calc. for $\mathrm{C}_{63} \mathrm{H}_{64} \mathrm{~N}_{2} \mathrm{O}_{25} \mathrm{~S}$ : C, 59.06; H, 5.03. Found: C, 59.27; $\mathrm{H}$, $5.11 \%)$.

\section{Isopropyl 2,3,4,6-tetra- $O$-benzyl-1-thio- $\beta$-D-galactopyranoside 18}

To a cold $\left(0{ }^{\circ} \mathrm{C}\right)$ solution of IPTG $(1.0 \mathrm{~g}, 4.20 \mathrm{mmol})$ in DMF $(10 \mathrm{~mL})$ were added $\mathrm{NaH}(50 \% ; 1.66 \mathrm{~g}, 34.6 \mathrm{mmol})$ and $\mathrm{BnBr}$ $(2.3 \mathrm{~mL}, 18.9 \mathrm{mmol})$ cautiously. The reaction mixture was stirred at room temperature for $6 \mathrm{~h}$, then poured into cold water, extracted with $\mathrm{CH}_{2} \mathrm{Cl}_{2}(2 \times 30 \mathrm{~mL})$, and the organic layer was dried over $\mathrm{Na}_{2} \mathrm{SO}_{4}$, and concentrated. Column chromatography $(5: 1$, petroleum ether-ethyl acetate) of the residue gave $18(2.1 \mathrm{~g}, 85 \%)$ as a syrup; $[\alpha]_{\mathrm{D}}^{25}-5\left(c 1, \mathrm{CHCl}_{3}\right) ;{ }^{1} \mathrm{H} \mathrm{NMR}$ $\delta 1.28,1.30\left[2 \mathrm{~d}, 6 \mathrm{H}, \mathrm{SCH}\left(\mathrm{CH}_{3}\right)_{2}\right], 3.14-3.23(\mathrm{~m}, 1 \mathrm{H}, \mathrm{SCH})$, 3.51-3.57 (m, 4 H, H-3, H-5, H-6a, H-6b), 3.77 (t, 1 H, $J=9.6$ $\mathrm{Hz}, \mathrm{H}-2), 3.91\left(\mathrm{~d}, 1 \mathrm{H}, J_{3,4} 2.7 \mathrm{~Hz}, \mathrm{H}-4\right), 4.35(\mathrm{~d}, 1 \mathrm{H}, J 11.4 \mathrm{~Hz}$ $\left.\mathrm{CH}_{2}\right), 4.42\left(\mathrm{~d}, 1 \mathrm{H}, J 11.4 \mathrm{~Hz}, \mathrm{CH}_{2}\right), 4.46\left(\mathrm{~d}, 1 \mathrm{H}, J_{1,2} 9.6 \mathrm{~Hz}\right.$ $\mathrm{H}-1), 4.58$ (d, $\left.1 \mathrm{H}, J 11.7 \mathrm{~Hz}, \mathrm{CH}_{2}\right), 4.69$ (s, $\left.2 \mathrm{H}, \mathrm{CH}_{2}\right), 4.73$ (d, $\left.1 \mathrm{H}, J 9.9 \mathrm{~Hz}, \mathrm{CH}_{2}\right), 4.85\left(\mathrm{~d}, 1 \mathrm{H}, J 10.2 \mathrm{~Hz}, \mathrm{CH}_{2}\right), 4.91(\mathrm{~d}, 1 \mathrm{H}$, $\left.J 11.7 \mathrm{~Hz}, \mathrm{CH}_{2}\right), 7.18-7.36(\mathrm{~m}, 20 \mathrm{H}, \mathrm{Ph})\left(\right.$ Calc. for $\mathrm{C}_{37} \mathrm{H}_{42} \mathrm{O}_{5} \mathrm{~S}$ : C, 74.22; H, 7.07. Found: C, 74.54; H, 6.96\%).

\section{Methyl $O$-[2,3,4,6-tetra- $O$-benzyl- $\alpha$-D-galactopyranosyl- $(1 \rightarrow 4)$ ]-3-O-benzoyl-2,6-di- $O$-benzyl- $\beta$-D-galacto- pyranoside 20}

To a solution of compound $\mathbf{1 8}(300 \mathrm{mg}, 0.5 \mathrm{mmol})$ and $\mathbf{1 9}^{7}$ (240 mg, $0.50 \mathrm{mmol})$ in anhydrous $\mathrm{CH}_{2} \mathrm{Cl}_{2}(6 \mathrm{~mL})$ were added NIS $(282 \mathrm{mg}, 1.25 \mathrm{mmol})$ and $\mathrm{Me}_{3} \operatorname{SiOTf}(27 \mu \mathrm{L}, 0.18 \mathrm{mmol})$ under $\mathrm{N}_{2}$ at $0{ }^{\circ} \mathrm{C}$. The mixture was stirred under these conditions for $2 \mathrm{~h}$, at which time TLC $(2: 1$, petroleum etherEtOAc) indicated that starting material $\mathbf{1 8}$ was consumed completely. The reaction mixture was neutralized with $\mathrm{Et}_{3} \mathrm{~N}$, then concentrated. Column chromatography $(5: 2$, petroleum ether-EtOAc) of the residue gave $\mathbf{2 0}(400 \mathrm{mg}, 80 \%)$ as a syrup; $[a]_{\mathrm{D}}^{25}+44\left(c 1, \mathrm{CHCl}_{3}\right) ;{ }^{1} \mathrm{H}$ NMR $\delta 3.08\left(\mathrm{dd}, 1 \mathrm{H}, J_{5,6 \mathrm{a}} 8.4, J_{6 \mathrm{a}, 6 \mathrm{~b}}\right.$ $5.4 \mathrm{~Hz}, \mathrm{H}-6 \mathrm{a}), 3.40$ (dd, $1 \mathrm{H}, J$ 9.6, $8.4 \mathrm{~Hz}, \mathrm{H}-5), 3.63$ (s, $3 \mathrm{H}$, $\mathrm{OCH}_{3}$ ), 3.67-3.83 (m, 4 H, H-2', H-5, H-6a, H-6b), 3.89 (dd, $\left.1 \mathrm{H}, J_{5,6 \mathrm{~b}} 9.6 \mathrm{~Hz}, \mathrm{H}-6 \mathrm{~b}\right), 3.99-4.03$ (m, $\left.2 \mathrm{H}, \mathrm{H}-2, \mathrm{H}-3^{\prime}\right), 4.04$ (s, $1 \mathrm{H}, \mathrm{H}-4), 4.11\left(\mathrm{~s}, 1 \mathrm{H}, \mathrm{H}-4^{\prime}\right), 4.26\left(\mathrm{~s}, 2 \mathrm{H}, \mathrm{CH}_{2}\right), 4.31(\mathrm{~s}, 2 \mathrm{H}$, $\left.\mathrm{CH}_{2}\right), 4.42\left(\mathrm{~d}, 1 \mathrm{H}, J_{1,2} 7.2 \mathrm{~Hz}, \mathrm{H}-1\right), 4.50(\mathrm{~d}, 1 \mathrm{H}, J 11.7 \mathrm{~Hz}$, $\left.\mathrm{CH}_{2}\right), 4.60\left(\mathrm{~d}, 1 \mathrm{H}, J 11.7 \mathrm{~Hz}, \mathrm{CH}_{2}\right), 4.61(\mathrm{~d}, 1 \mathrm{H}, J 11.7 \mathrm{~Hz}$, $\left.\mathrm{CH}_{2}\right), 4.75\left(\mathrm{~s}, 2 \mathrm{H}, \mathrm{CH}_{2}\right), 4.80\left(\mathrm{~d}, 1 \mathrm{H}, J 11.7 \mathrm{~Hz}, \mathrm{CH}_{2}\right), 4.82(\mathrm{~d}$, $\left.1 \mathrm{H}, J 11.7 \mathrm{~Hz}, \mathrm{CH}_{2}\right), 4.89$ (d, $\left.1 \mathrm{H}, J 11.4 \mathrm{~Hz}, \mathrm{CH}_{2}\right), 4.91(\mathrm{~s}, 1 \mathrm{H}$, H-1'), $5.11\left(\mathrm{dd}, 1 \mathrm{H}, J_{2,3} 10.2 \mathrm{~Hz}, J_{3,4} 3.0 \mathrm{~Hz}, \mathrm{H}-3\right), 7.06-8.09$ $(\mathrm{m}, 35 \mathrm{H}, \mathrm{Ph})$ [Calc. for $\mathrm{C}_{62} \mathrm{H}_{64} \mathrm{NaO}_{12}(\mathrm{M}+\mathrm{Na})^{+}:$1023.4. Found: $\left.m / z, 1023.1(\mathrm{M}+\mathrm{Na})^{+}\right]\left(\right.$Calc. for $\mathrm{C}_{62} \mathrm{H}_{64} \mathrm{O}_{12}: \mathrm{C}, 74.38$; H, 6.44. Found: C, 74.70; H, 6.32\%).

\section{Phenyl $O$-[2,3,4,6-tetra- $O$-benzyl- $\alpha$-D-galactopyranosyl- $(1 \longrightarrow 3)$ ]-2,6-di- $O$-benzoyl-1-thio- $\beta$-D-galacto- pyranoside 22}

To a solution of compound $\mathbf{1 8}(145 \mathrm{mg}, 0.24 \mathrm{mmol})$ and $\mathbf{2 1}$ $(97 \mathrm{mg}, 0.2 \mathrm{mmol})$ in anhydrous $\mathrm{Et}_{2} \mathrm{O}-\mathrm{CH}_{2} \mathrm{Cl}_{2}(1: 1 ; 3 \mathrm{~mL})$ were added NIS $(63 \mathrm{mg}, 0.28 \mathrm{mmol})$ and $\mathrm{Me}_{3} \mathrm{SiOTf}(13 \mu \mathrm{L}$,
$0.07 \mathrm{mmol}$ ) under $\mathrm{N}_{2}$ at $-42{ }^{\circ} \mathrm{C}$. The mixture was stirred under these conditions for $1 \mathrm{~h}$, at which time TLC $(2: 1$, petroleum ether-EtOAc) indicated that starting material 18 was consumed completely. The reaction mixture was neutralized with $\mathrm{Et}_{3} \mathrm{~N}$, then concentrated. Column chromatography $(4: 1$, petroleum ether-EtOAc) of the residue gave the pure product 22 (48 $\mathrm{mg}$, $62 \%$ based on $60 \mathrm{mg}$ of recovered acceptor 21) as a syrup; $[a]_{\mathrm{D}}^{25}+5\left(c 1, \mathrm{CHCl}_{3}\right) ;{ }^{1} \mathrm{H}$ NMR $\delta 2.85\left(\mathrm{dd}, 1 \mathrm{H}, J_{5,6 \mathrm{a}} 8.4 \mathrm{~Hz}\right.$, $\left.J_{6 \mathrm{~b}, 6 \mathrm{a}} 5.6 \mathrm{~Hz}, \mathrm{H}-6 \mathrm{a}^{\prime}\right), 3.28\left(\mathrm{t}, 1 \mathrm{H}, J_{5,6 \mathrm{~b}} 8.4 \mathrm{~Hz}, \mathrm{H}-5^{\prime}\right), 3.76$ (dd, $\left.1 \mathrm{H}, \mathrm{H}-6 \mathrm{~b}^{\prime}\right), 3.74-3.99$ (m, $\left.9 \mathrm{H}\right), 4.01$ (dd, $1 \mathrm{H}, J 10,2.8 \mathrm{~Hz}$, H-2'), 4.39-4.89 (m, $\left.8 \mathrm{H}, 4 \times \mathrm{PhCH}_{2}\right), 4.77$ (d, $1 \mathrm{H}, J 2.8 \mathrm{~Hz}$, H-1'), $4.80(\mathrm{~d}, 1 \mathrm{H}, J 10 \mathrm{~Hz}, \mathrm{H}-1), 5.52(\mathrm{t}, 1 \mathrm{H}, J 10 \mathrm{~Hz}$, $\mathrm{H}-2)$, 7.04-7.63 (m, $30 \mathrm{H}, \mathrm{Ph}), 8.06-8.92(\mathrm{~m}, 5 \mathrm{H}, \mathrm{Ph})$ (Calc. for $\mathrm{C}_{60} \mathrm{H}_{58} \mathrm{O}_{12} \mathrm{~S}: \mathrm{C}, 71.84 ; \mathrm{H}, 5.83$. Found: $\mathrm{C}, 72.03 ; \mathrm{H}, 5.92 \%$ ).

Phenyl $O$-[2,3,4,6-tetra- $O$-benzyl-D-galactopyranosyl-( $1 \longrightarrow 6)]$ 2,3,4-tri- $O$-benzoyl-1-thio- $\beta$-D-galactopyranoside 24

To a mixture of compound $\mathbf{1 8}(470 \mathrm{mg}, 0.786 \mathrm{mmol})$ and $\mathbf{2 3}$ $(330 \mathrm{mg}, 0.56 \mathrm{mmol})$ in anhydrous $\mathrm{Et}_{2} \mathrm{O}(5 \mathrm{~mL})$ were added NIS (174 mg, $0.78 \mathrm{mmol})$ and $\mathrm{Me}_{3} \operatorname{SiOTf}(7 \mu \mathrm{L}, 0.04 \mathrm{mmol})$ under $\mathrm{N}_{2}$ at $-42{ }^{\circ} \mathrm{C}$. The mixture was stirred under these conditions for $1 \mathrm{~h}$, at which time TLC ( $: 1$, petroleum ether-EtOAc) indicated that starting material was consumed completely. The reaction mixture was neutralized with $\mathrm{Et}_{3} \mathrm{~N}$, then concentrated. Column chromatography $(2: 1$, petroleum ether-EtOAc) of the residue gave $24(600 \mathrm{mg}, 96 \% ; \beta: \alpha=1: 2)$ as a syrup; ${ }^{1} \mathrm{H}$ NMR $\delta 3.47-3.52(\mathrm{~m}, 3 \mathrm{H}), 3.55(\mathrm{dd}, 0.33 \mathrm{H}), 3.68$ (dd, $0.33 \mathrm{H}), 3.81-$ $4.05(\mathrm{~m}, 4.33 \mathrm{H}), 4.07\left(\mathrm{t}, 0.33 \mathrm{H}, J 9.8 \mathrm{~Hz}, \mathrm{H}-2^{\prime}\right.$ of $\beta$ isomer $)$, $4.31-5.00(\mathrm{~m}, 10.67 \mathrm{H}), 5.51(\mathrm{dd}, 0.67 \mathrm{H}, J 9.8,3.2 \mathrm{~Hz}, \mathrm{H}-3$ of $\alpha$ isomer), 5.55 (dd, $0.33 \mathrm{H}, J 9.8,3.2 \mathrm{~Hz}, \mathrm{H}-3$ of $\beta$ isomer), 5.69 (t, $0.33 \mathrm{H}, J 9.8 \mathrm{~Hz}, \mathrm{H}-2$ of $\beta$ isomer), $5.70(\mathrm{t}, 0.67 \mathrm{H}, J 9.8 \mathrm{~Hz}$, $\mathrm{H}-2$ of $\alpha$ isomer), $5.88(\mathrm{~d}, 0.67 \mathrm{H}, J 3.1 \mathrm{~Hz}, \mathrm{H}-4$ of $\alpha$ isomer), $5.91(\mathrm{~d}, 0.33 \mathrm{H}, J 3.1 \mathrm{~Hz}, \mathrm{H}-4$ of $\beta$ isomer), $7.22-7.97(\mathrm{~m}, 40 \mathrm{H}$, $\mathrm{Ph}$ ) (Calc. for $\mathrm{C}_{67} \mathrm{H}_{62} \mathrm{O}_{13} \mathrm{~S}$ : C, 72.68; H, 5.64. Found: C, 72.91; $\mathrm{H}, 5.57 \%)$.

\section{Phenyl 2,3-di- $O$-benzoyl-6- $O$-benzyl-1-thio- $\beta$-D-galacto- pyranoside 25}

$1.04 \mathrm{~g}$ of phenyl 4,6- $O$-benzylidene-1-thio- $\beta$-D-galactopyrano$\operatorname{side}^{9}(2.86 \mathrm{mmol})$ was dissolved in $10 \mathrm{~mL}$ of pyridine, then premixed benzoyl chloride $(2.2 \mathrm{~mL}, 18.5 \mathrm{mmol})$ and pyridine $(3 \mathrm{~mL})$ was added; the mixture was stirred vigorously at $50{ }^{\circ} \mathrm{C}$ overnight, and then poured into ice-cold water, and extracted with $\mathrm{CH}_{2} \mathrm{Cl}_{2}(2 \times 30 \mathrm{~mL})$. The organic phase was concentrated to dryness by repeated co-evaporation with toluene. The residue was subjected to column chromatography on silica gel with petroleum ether-EtOAc as eluent $(2: 1)$ to give phenyl 2,3di- $O$-benzoyl-4,6- $O$-benzylidene-1-thio- $\beta$-D-galactopyranoside $(1.5 \mathrm{~g}, 92 \%) ;[\alpha]_{\mathrm{D}}^{25}+6\left(c 1, \mathrm{CHCl}_{3}\right) ;{ }^{1} \mathrm{H}$ NMR $\delta 3.76$ (br s, $1 \mathrm{H}$, $\mathrm{H}-5), 4.10\left(\mathrm{dd}, 1 \mathrm{H}, J_{5,6 \mathrm{a}} 1.2 \mathrm{~Hz}, J_{6 \mathrm{a}, 6 \mathrm{~b}} 12.4 \mathrm{~Hz}, \mathrm{H}-6 \mathrm{a}\right), 4,45$ (dd, $\left.1 \mathrm{H}, J_{5,6 \mathrm{~b}} 1 \mathrm{~Hz}, J_{6 \mathrm{a}, 6 \mathrm{~b}} 12.4 \mathrm{~Hz}, \mathrm{H}-6 \mathrm{~b}\right), 4.60\left(\mathrm{~d}, 1 \mathrm{H}, J_{3,4} 3.2 \mathrm{~Hz}\right.$, $\mathrm{H}-4), 4.97$ (d, $\left.1 \mathrm{H}, J_{1,2} 9.8 \mathrm{~Hz}, \mathrm{H}-1\right), 5.36$ (dd, $1 \mathrm{H}, J 9.8,3.2 \mathrm{~Hz}$, $\mathrm{H}-3), 5.51$ (s, 1 H, PhCH), 5.81 (t, $1 \mathrm{H}, J 9.8 \mathrm{~Hz}, \mathrm{H}-2), 7.24$ $7.98(\mathrm{~m}, 20 \mathrm{H}, \mathrm{Ph})$.

$129 \mathrm{mg}$ of phenyl 2,3-di- $O$-benzoyl-4,6- $O$-benzylidene- $\beta$-Dgalactopyranoside $(0.225 \mathrm{mmol})$ and $311 \mathrm{mg}$ of sodium cyanoborohydride $(4.5 \mathrm{mmol})$ were dissolved in $3 \mathrm{~mL}$ of dry THF (distilled from sodium), then $5 \mathrm{~mL}$ of hydrogen chloride saturated diethyl ether was added with vigorous stirring at room temperature. A second portion of hydrogen chloride in diethyl ether $(2 \mathrm{~mL})$ was added dropwise during $1.5 \mathrm{~h}$ until evolution of the gas ceased. The reaction mixture was diluted with $\mathrm{CH}_{2} \mathrm{Cl}_{2}(50 \mathrm{~mL})$, then washed with saturated aq. $\mathrm{NaHCO}_{3}$. The organic phase was dried over $\mathrm{Na}_{2} \mathrm{SO}_{4}$ and concentrated to dryness. The residue was subjected to column chromatography on silica gel with petroleum ether-EtOAc as eluent $(4: 1)$ to give $25(106 \mathrm{mg}, 82 \%)$ as a syrup; $[\alpha]_{\mathrm{D}}^{25}+85(c 1$, $\mathrm{CHCl}_{3}$ ); ${ }^{1} \mathrm{H}$ NMR $\delta 2.72$ (br s, $\left.1 \mathrm{H}, 4-\mathrm{OH}\right), 3.84-3.92(\mathrm{~m}, 3 \mathrm{H}$, $\left.\mathrm{H}-5, \mathrm{H}_{2}-6\right), 4.42\left(\mathrm{~d}, 1 \mathrm{H}, J_{3,4} 2.7 \mathrm{~Hz}, \mathrm{H}-4\right), 4.60$ (2 d, $2 \mathrm{H}, J 12.0$ 
$\mathrm{Hz}, \mathrm{PhCH}_{2}$ ), 4.94 (d, $\left.1 \mathrm{H}, J_{1,2} 9.8 \mathrm{~Hz}, \mathrm{H}-1\right), 5.32$ (dd, $1 \mathrm{H}, J 9.8$, $3.0 \mathrm{~Hz}, \mathrm{H}-3), 5.79$ (t, $1 \mathrm{H}, J 9.8 \mathrm{~Hz}, \mathrm{H}-2), 7.25-7.98$ (m, $20 \mathrm{H}$, $\mathrm{Ph}$ ) (Calc. for $\mathrm{C}_{33} \mathrm{H}_{30} \mathrm{O}_{7} \mathrm{~S}: \mathrm{C}, 69.46 ; \mathrm{H}, 5.30$. Found: C, 69.71; H, $5.39 \%)$.

\section{Acknowledgements}

This work was supported by National Nature Science Foundation of China (Projects 29972053 and 39970179).

\section{References}

1 (a) E. E. Simanek, G. J. McGarvey, J. A. Jablonowski and
C.-H. Wong, Chem. Rev., 1998, 98, 833; (b) R. A. Dwek, Chem. Rev., 1996, 96, 683.

2 G.-J. Boons and K. J. Hale, Organic Synthesis with Carbohydrates, Sheffield Academic Press, England, 2000.

3 Y. Du, M. Zhang and F. Kong, Org. Lett., 2000, 2, 3797.

4 (a) L. J. Huang, Y. Lin and G. Y. Tian, Acta Pharma. Sin., 1998, 33, 512; (b) L. J. Huang, G. Y. Tian and G. Z. Ji, J. Asian Nat. Prod. Res., $1999,1,259$.

5 (a) Y. Du, J. Lin and R. J. Linhardt, J. Carbohydr. Chem., 1997, 16, 1327; (b) F. Belot and J. C. Jacquint, Carbohydr. Res., 1996, 290, 79. 6 Y. Du and F. Kong, J. Carbohydr. Chem., 1995, 14, 341.

7 M. Zhang, MSc dissertation, Chinese Academy of Sciences, 2001.

8 K. Toshima and K. Tatsuta, Chem. Rev., 1993, 93, 1503.

9 A. Fernandez-Mayoralas, A. Marra, M. Trumtel, A. Veyrières and P. Sinaÿ, Tetrahedron Lett., 1989, 30, 2537. 